\title{
Arthropods associated with pig carrion in two vegetation profiles of Cerrado in the State of Minas Gerais, Brazil
}

\author{
Thiago Augusto Rosa ${ }^{1,2}$, Micaela L. Y. Babata ${ }^{1}$, Carina Mara de Souza ${ }^{1}$, Danielle de Sousa ${ }^{1}$, \\ Cátia A. de Mello-Patiu ${ }^{3}$, Fernando Z. Vaz-de-Mello ${ }^{4} \&$ Júlio Mendes ${ }^{1}$
}

\begin{abstract}
${ }^{1}$ Laboratório de Entomologia Médica e Veterinária, Instituto de Ciências Biomédicas, Universidade Federal de Uberlândia, Campus Umuarama, Caixa Postal 593, 38405-302 Uberlândia-MG, Brazil. jmendes@ufu.br

${ }^{2}$ Laboratório de EcoZoologia, Faculdade de Ciências Integradas do Pontal/FACIP, Av. José João Dib 2545, Bairro Progresso, $38302-000$ Ituiutaba-MG, Brazil. thi_aug@pontal.ufu.br

${ }^{3}$ Departamento de Entomologia, Universidade Federal do Rio de Janeiro, Museu Nacional, Quinta da Boa Vista S/N, São Cristóvão, $20940-040$ Rio de Janeiro-RJ, Brazil. catiapatiu@oi.com.br

${ }^{4}$ Departamento de Biologia e Zoologia, Instituto de Biociências, Universidade Federal de Mato Grosso, Av. Fernando Corrêa da Costa 2367, Boa Esperança, 78060-900 Cuiabá-MT, Brasil. vazdemello@gmail.com
\end{abstract}

\begin{abstract}
Arthropods associated with pig carrion in two vegetation profiles of Cerrado in the State of Minas Gerais, Brazil. Forensic Entomology research has been concentrated in only a few localities of the "Cerrado" vegetation, the Brazilian Savannah. The present study had, as its objective, an examination of the diversity of arthropod fauna associated with the carcasses of Sus scrofa (Linnaeus) in this biome. The study was conducted during the dry and humid periods in two Cerrado vegetation profiles of the State of Minas Gerais. The decaying process was slower and greater quantities of arthropods were collected during the dry period. Insects represented $99 \%$ of 161,116 arthropods collected. The majority of these were Diptera $(80.2 \%)$ and Coleoptera $(8.8 \%)$. The entomofauna belong to 85 families and at least 212 species. Diptera were represented by 31 families and at least 132 species. Sarcophagidae (Diptera) and Scarabaeidae (Coleoptera) were the richest groups. Oxysarcodexia (Sarcophagidae) presented the largest number of attracted species, however none of these species bred in the carcasses. The Coleoptera collected belong to at least 50 species of 21 families. Among these species, Dermestes maculatus and Necrobia rufipes were observed breeding in the carcasses. This study showed species with potential importance for estimating the postmortem interval (PMI), indicative of seasonal and environmental type located.
\end{abstract}

KEYWORDS. Coleoptera; Diptera; ecological succession; insect diversity.

RESUMO. Artrópodes associados a carcaças de suínos em dois perfis de vegetação de Cerrado no Estado de Minas Gerais, Brasil. Estudos de entomologia forense em área de Cerrado ainda são escassos no Brasil. Este trabalho teve como objetivo estudar a riqueza da artropodofauna associada a carcaças de suínos domésticos em decomposição. O estudo foi conduzido em dois perfis de Cerrado, Cerrado senso stricto e Campo sujo, durante dois períodos do ano, seco e úmido, em Uberlândia, MG, Brasil. Insetos representaram $99 \%$ dos 161.116 artrópodes coletados, sendo representados majoritariamente por dípteros $(80,2 \%)$ e coleópteros $(8,8 \%)$. A entomofauna era pertencente a 85 famílias e pelo menos 212 espécies. Os dípteros foram representados por 31 famílias e pelo menos 132 espécies. Os Sarcophagidae (Diptera) e Scarabaeidae (Coleoptera) foram os grupos com maior riqueza de espécies. Oxysarcodexia (Sarcophagidae) apresentou o maior número de espécies atraídas. Os coleópteros coletados pertenciam a pelo menos 21 famílias e 50 espécies, dentre as quais Dermestes maculatus e Necrobia rufipes foram observadas criando-se nas carcaças. Este estudo apresenta várias espécies com potencial importância para estimar o Intervalo Pós-Morte (IPM), e indicativas de sazonalidade e de ambiente localizado.

PALAVRAS-CHAVE. Coleoptera; Diptera; diversidade entomológica; sucessão entomológica.

The importance of entomofauna in the decomposition of animal carcasses is well known. While a vast number of insects use these substrates as food sources for their immature and/or adults forms, others use them as habitats for trophic interaction with other species (Hanski 1987). Adequate knowledge of a natural area also requires studies of the fauna associated with carrion (Turchetto \& Vanin 2004). The potential of the insects as forensic indicators has stimulated detailed research regarding the role of fauna in carcass decomposition in various regions and countries (Carvalho \& Linhares 2001; Centeno et al. 2002; Oliveira-Costa \& MelloPatiu 2004; Kimberly et al. 2005; Velásquez 2008). These studies have reported great diversity in the entomofauna found in animal carcasses during the decomposition process and the preference of species for specific stages of decomposition, types of environments and seasons (Campobasso et al 2001; Turchetto \& Vanin 2004; Archer et al. 2005). Species that present such peculiarities are useful as forensic indicators in their respective study regions.

There is increasing interest in Medico-legal Forensic Entomology in Brazil and, as a result, the number of papers published in this area has increased (Carvalho et al. 2000; Carvalho \& Linhares 2001; Oliveira-Costa \& Mello-Patiu 2004; Carvalho et al. 2004; Thyssen et al. 2005; Barros et al. 
2008; Barbosa et al. 2009). Brazil, a country of continental dimensions, presents notable variation in climate and vegetation among its regions. Studies of forensic entomology in the Cerrado are rare and restricted to only a few localities in this country (Marchiori et al. 2000; Barros et al. 2008).

In a recent study, Rosa et al. (2009) presented detailed information regarding the Diptera found in pig carcasses in this type of vegetation, in Uberlândia, State of Minas Gerais. The present work presents the diversity of entomofauna associated with pig carcasses in two Cerrado profiles in this same region, during the humid and dry seasons.

\section{MATERIAL AND METHODS}

The experiments were conducted in a natural area of Cerrado (518,98319 S; 048,29723 W), situated seven kilometers south of the urban perimeter of the city of Uberlândia, State of Minas Gerais, Brazil. The study was conducted during two periods of the year: first in the dry period of 2005 and the second in the humid period of 2006 and in two vegetation profiles, "Campo sujo" and "Cerrado stricto sensu". "Campo sujo" presents predominantly herbaceous, sub-shrub, and sparse shrub and arboreal vegetation. "Cerrado stricto sensu" is composed predominantly of shrubs and trees with contorted forms, three to five meters in height (Ribeiro et al. 1983). In each period, four carcasses of domestic pigs (Sus scrofa Linnaeus) weighing $10 \pm 0.5 \mathrm{~kg}$ were exposed, two in each of the vegetation profiles, 150 meters from each other. The distance between sites in each of the two vegetation profiles was approximately two kilometers. Removable trays two $\mathrm{cm}$ deep containing sawdust were placed on the bottom of the cages to retain ambulatory and immature insects. A pyramidal support of iron bars covered with a thin cotton fabric for the capture of winged insects attracted to the carcass was installed above the cages. A thermo-hygrometer was located in each area to obtain daily temperature and humidity readings during the decomposition process. Precipitation levels were obtained from the weather station of the Federal University of Uberlândia, located 10 and $12 \mathrm{~km}$ from the study areas.

The sawdust in the trays were replaced daily and taken to the laboratory for analysis. Winged insects that had been captured were also collected daily, using collector flasks and insect nets. The sawdust with immature specimens (post-feeding larvae) was maintained in the laboratory until adult emergence. The decomposition process was divided according to the classification of Bornemissza (1957): 1) initial decay, 2) putrefaction, 3) black putrefaction, 4) butyric fermentation and 5) dry decay. Details of the procedures adopted are described in Rosa et al. (2009).

\section{RESULTS}

Decomposition in the first phase of the experiment lasted 68 days, from July 22 to September 28, 2005. This period had average daily temperatures of $24.87^{\circ} \mathrm{C}\left( \pm 3.3^{\circ} \mathrm{C}\right)$, rela- tive humidity of $52.7 \%( \pm 6.1 \%)$ and a total precipitation of $50.63 \mathrm{~mm}^{3}$, characterizing the dry period. The second phase was undertaken from January 27 to March 4, 2006, totaling 37 days. This period had average temperatures of $25.7^{\circ} \mathrm{C}$ $\left( \pm 2.6^{\circ} \mathrm{C}\right)$, relative humidity of $60.2 \%( \pm 5.3 \%)$ and a total of $302 \mathrm{~mm}^{3}$ of precipitation, thus characterizing a warmer and more humid period.

The first two stages of decay had respective durations of one and three days in both periods of the year. The third stage was five days in duration during the dry period and three days in the humid one. The fourth and fifth stages of decay presented durations of 18 and 41 days during the dry period and 13 and 18 days in the humid period (data from Rosa et al. 2009).

Carcasses were visited mainly by Calliphoridae, Sarcophagidae, Muscidae and Fanniidae during the first three stages of decay. As the decaying process advanced (fourth and fifth stages), these dipterans were replaced by Stratiomyidae (Diptera), Dermestidae and Cleridae (Coleoptera).

Both areas presented a great diversity of insects during the two study periods. A total of 161,116 arthropods were collected and almost all of them (99\%) were insects belonging to 85 families and at least 212 species. Diptera and Coleoptera represented respectively $80.2 \%$ and $8.8 \%$ of all collected entomofauna. Many other groups of insects were also collected. Heteroptera, Hymenoptera and Lepidoptera were most representative among the other groups (Table I).

Diptera were represented by 31 families and at least 132 species. The Sarcophagidae were notable due to their diversity of species, representing $67.1 \%$ of total insects collected and $38 \%$ of species. Coleoptera were the second in species diversity with $8.7 \%$ of relative frequency and $28 \%$ of all attracted species. Scarabaeidae and Staphylinidae were notable due to their great diversity among the coleopterans (Table I). Two Coleoptera species used the carcasses as breeding substrate (Table I). Data on the Diptera reared in the carcasses are presented in Rosa et al. (2009).

Oxysarcodexia (Diptera, Sarcophagidae) demonstrated the greatest diversity of species attracted to the carcasses (Table I). Chrysomya albiceps Wiedemann was the most abundant among Calliphoridae (Table I). Cochliomyia macellaria and Chrysomya putoria Wiedemann were other Calliphoridae that presented abundance in the carcasses (Table I). Ophyra aenescens Wiedemann was the Muscidae most observed and was collected during the two periods of the year. Musca domestica Linnaeus and Stomoxys calcitrans Linnaeus also were collected during both periods (Table I). In addition to the significant participation of Diptera species of the above mentioned families in the carcass decomposition, the less frequent presence of Fanniidae, Stratiomyidae and Phoridae (Diptera) was also observed (Table I).

The study registered the occurrence of Sarcophagidae Blaesoxipha (Acantodotheca) minensis Lopes \& Downs, Helicobia morionella Aldrich, Oxysarcodexia culmiforceps Aldrich and Tricharaea (Sarcophagula) ramirezi Lopes, in 
Table I. Absolute (AF) and relative (\%) frequencies of arthropods attracted to pig carrion ( Sus scrofa L.) during dry period of 2005 and the humid period of 2006 in Uberlândia, State of Minas Gerais. * Species reared from the carcasses (from Rosa et al. 2009).

\begin{tabular}{|c|c|c|c|c|c|c|}
\hline \multirow{2}{*}{ Taxa } & \multicolumn{2}{|c|}{ Dry period } & \multicolumn{2}{|c|}{ Humid period } & \multicolumn{2}{|c|}{ Total } \\
\hline & $\mathrm{AF}$ & $\%$ & $\mathrm{AF}$ & $\%$ & $\mathrm{AF}$ & $\%$ \\
\hline \multicolumn{7}{|l|}{ Diptera } \\
\hline \multicolumn{7}{|l|}{ Sarcophagidae } \\
\hline Argoravinia rufiventris (Wiedemann, 1830) & 21 & 0.03 & 5 & 0.07 & 26 & 0.03 \\
\hline Blaesoxipha (Acantodotheca) lanei (Lopes, 1938) & 1,297 & 1.62 & 233 & 3.41 & 1,530 & 1.76 \\
\hline Blaesoxipha (Acantodotheca) minensis (Lopes \& Downs, 1951) & 25 & 0.03 & 0 & 0.00 & 25 & 0.03 \\
\hline Dexosarcophaga carvalhoi (Lopes, 1980) & 5,234 & 6.55 & 238 & 3.48 & 5,47 & 6.31 \\
\hline Dexosarcophaga paulistana (Lopes, 1982) & 1 & 0.00 & 0 & 0.00 & 1 & 0.00 \\
\hline Dexosarcophaga spp. & 229 & 0.29 & 165 & 2.42 & 394 & 0.45 \\
\hline Dexosarcophaga transita (Townsend, 1917) & 403 & 0.50 & 5 & 0.07 & 408 & 0.47 \\
\hline Helicobia aurescens (Townsend, 1927) & 126 & 0.16 & 47 & 0.69 & 173 & 0.20 \\
\hline Helicobia borgmeieri Lopes, 1939 & 3 & 0.01 & 0 & 0.00 & 3 & 0.00 \\
\hline Helicobia morionella (Aldrich, 1930) & 35 & 0.04 & 0 & 0.00 & 35 & 0.04 \\
\hline Helicobia pilifera Lopes, 1939 & 67 & 0.08 & 6 & 0.09 & 73 & 0.08 \\
\hline Helicobia rapax (Walker, 1842) & 161 & 0.20 & 26 & 0.38 & 187 & 0.22 \\
\hline Microcerella erythropyza (Lopes, 1936) & 19 & 0.02 & 0 & 0.00 & 19 & 0.02 \\
\hline Oxysarcodexia admixta (Lopes, 1933) & 138 & 0.17 & 3 & 0.04 & 141 & 0.16 \\
\hline Oxysarcodexia amorosa (Skiner,1868) & 3 & 0.00 & 0 & 0.00 & 3 & 0.00 \\
\hline Oxysarcodexia angrensis (Lopes,1933) & 333 & 0.42 & 8 & 0.12 & 341 & 0.39 \\
\hline Oxysarcodexia aura (Hall, 1937) & 667 & 0.83 & 5 & 0.07 & 672 & 0.78 \\
\hline Oxysarcodexia avuncula (Lopes, 1932) & 11,588 & 14.50 & 305 & 4.47 & 11,893 & 13.70 \\
\hline Oxysarcodexia carvalhoi Lopes, 1946 & 29 & 0.07 & 4 & 0.06 & 33 & 0.04 \\
\hline Oxysarcodexia culmiforceps Dodge, 1966 & 10 & 0.01 & 0 & 0.00 & 10 & 0.01 \\
\hline Oxysarcodexia diana (Lopes, 1933) & 891 & 1.12 & 40 & 0.59 & 931 & 1.07 \\
\hline Oxysarcodexia eberti Lopes \& Tibana, 1987 & 30 & 0.04 & 2 & 0.03 & 32 & 0.04 \\
\hline Oxysarcodexia fluminensis Lopes, 1946 & 253 & 0.32 & 12 & 0.18 & 265 & 0.31 \\
\hline Oxysarcodexia modesta Lopes, 1946 & 1 & 0.00 & 0 & 0.00 & 1 & 0.00 \\
\hline Oxysarcodexia paulistanensis (Mattos, 1919) & 32 & 0.04 & 1 & 0.02 & 33 & 0.04 \\
\hline Oxysarcodexia riograndensis Lopes, 1946 & 5 & 0.01 & 0 & 0.00 & 5 & 0.01 \\
\hline Oxysarcodexia simplicoides (Lopes, 1933) & 388 & 0.48 & 41 & 0.60 & 429 & 0.49 \\
\hline Oxysarcodexia spp. & 17 & 0.02 & 3 & 0.04 & 20 & 0.02 \\
\hline Oxysarcodexia terminalis (Wiedemann, 1830) & 1 & 0.00 & 0 & 0.00 & 1 & 0.0 \\
\hline Oxysarcodexia thornax (Walker, 1849) & 37,557 & 46.98 & 2,972 & 43.51 & 40,529 & 46.71 \\
\hline Oxysarcodexia xanthosoma (Aldrich, 1916) & 3 & 0.01 & 0 & 0.00 & 3 & 0.00 \\
\hline Peckia (Euboettcheria) anguilla (Curran \& Walley, 1934) & 132 & 0.17 & 24 & 0.35 & 156 & 0.18 \\
\hline Peckia (Euboettcheria) australis (Townsend, 1927) & 2 & 0.01 & 0 & 0.00 & 2 & 0.00 \\
\hline Peckia (Euboettcheria) collusor (Curran \& Walley, 1934) & 264 & 0.33 & 25 & 0.37 & 289 & 0.33 \\
\hline Peckia (Euboettcheria) florencioi (Prado \& Fonseca, 1932) & 89 & 0.11 & 21 & 0.31 & 110 & 0.13 \\
\hline Peckia (Pattonella) intermutans (Walker, 1861) * & 35 & 0.04 & 10 & 0.15 & 45 & 0.05 \\
\hline Peckia (Peckia) chrysostoma (Wiedemann, 1830) & 80 & 0.10 & 5 & 0.07 & 85 & 0.10 \\
\hline Peckia (Peckia) pexata (Wulp, 1895) & 73 & 0.09 & 2 & 0.03 & 75 & 0.09 \\
\hline Peckia (Squamatodes) ingens (Walker, 1849) & 6 & 0.01 & 2 & 0.03 & 8 & 0.01 \\
\hline Peckia (Squamatodes) trivittata (Curran, 1927) * & 197 & 0.25 & 98 & 1.44 & 295 & 0.34 \\
\hline Ravinia advena (Walker, 1853) & 360 & 0.45 & 52 & 0.76 & 412 & 0.48 \\
\hline Ravinia belforti (Prado \& Fonseca 1932) & 1,910 & 2.39 & 95 & 1.39 & 2,005 & 2.31 \\
\hline Sarcodexia lambens (Wiedemann, 1830) * & 1,035 & 1.29 & 331 & 4.85 & 1,366 & 1.57 \\
\hline Sarconeiva fimbriata (Aldrich, 1916) & 1 & 0.00 & 0 & 0.00 & 1 & 0.00 \\
\hline Sarcophaga (Lipoptilocnema) crispula (Lopes, 1938) & 124 & 0.16 & 2 & 0.03 & 126 & 0.15 \\
\hline
\end{tabular}


Table I. Continued.

\begin{tabular}{|c|c|c|c|c|c|c|}
\hline \multirow{2}{*}{ Taxa } & \multicolumn{2}{|c|}{ Dry period } & \multicolumn{2}{|c|}{ Humid period } & \multicolumn{2}{|c|}{ Total } \\
\hline & $\mathrm{AF}$ & $\%$ & $\mathrm{AF}$ & $\%$ & $\mathrm{AF}$ & $\%$ \\
\hline Titanogrypa (Cucullomyia) larvicida (Lopes, 1935) & 81 & 0.10 & 5 & 0.07 & 86 & 0.10 \\
\hline Tricharaea (Sarcophagula) occidua (Fabricius, 1794) & 4,602 & 5.76 & 1,606 & 3.51 & 6,208 & 7.16 \\
\hline Tricharaea (Sarcophagula) ramirezi (Lopes, 1990) & 28 & 0.04 & 0 & 0.00 & 28 & 0.03 \\
\hline Sarcophagidae spp. & 11,348 & 14.20 & 431 & 6.31 & 11,779 & 13.60 \\
\hline Total Sarcophagidae & 79,934 & 100.00 & 6,830 & 100.00 & 86,764 & 100.00 \\
\hline \multicolumn{7}{|l|}{ Muscidae } \\
\hline Atherigona orientalis (Schiner, 1868) & 1,007 & 16.74 & 35 & 2.17 & 1,042 & 13.65 \\
\hline Biopyrellia bipuncta (Wiedemann, 1830) & 855 & 14.21 & 1 & 0.06 & 856 & 11.22 \\
\hline Brontaea debilis (Williston, 1896) & 130 & 2.16 & 5 & 0.31 & 135 & 1.77 \\
\hline Brontaea spp. & 0 & 0.00 & 3 & 0.19 & 3 & 0.04 \\
\hline Cyrtoneurina sp. & 0 & 0.00 & 11 & 0.68 & 11 & 0.14 \\
\hline Cyrtoneuropsis conspersa (Stein, 1911) & 1 & 0.02 & 0 & 0.00 & 1 & 0.01 \\
\hline Cyrtoneuropsis rescita (Walker, 1861). & 453 & 7.53 & 241 & 14.91 & 694 & 9.09 \\
\hline Graphomya maculata (Scopoli, 1763) & 29 & 0.48 & 6 & 0.37 & 35 & 0.46 \\
\hline Myospila sp. & 2 & 0.03 & 0 & 0.00 & 2 & 0.03 \\
\hline Morellia ochricornis (Wiedemann, 1830) & 35 & 0.58 & 1 & 0.06 & 36 & 0.47 \\
\hline Musca domestica (Linnaeus, 1758)* & 1,693 & 28.14 & 234 & 14.48 & 1,927 & 25.25 \\
\hline Ophyra chalcogaster (Wiedemann, 1824) & 12 & 0.20 & 1 & 0.06 & 13 & 0.17 \\
\hline Ophyra aenescens (Wiedemann, 1830)* & 1,517 & 0.25 & 1,020 & 0.63 & 2,537 & 0.33 \\
\hline Pseudoptilolepis nigripoda (Snyder, 1949) & 0 & 0.00 & 14 & 0.87 & 14 & 0.18 \\
\hline Sarcopromusca pruna (Shannon \& Del Ponte, 1926) & 268 & 4.45 & 5 & 0.31 & 273 & 3.58 \\
\hline Stomoxys calcitrans (Linnaeus, 1758) * & 12 & 0.20 & 39 & 2.41 & 51 & 0.67 \\
\hline Synthesiomyia nudiseta (Wulp, 1883) & 2 & 0.03 & 0 & 0.00 & 2 & 0.03 \\
\hline Total Muscidae & 6,016 & 100.00 & 1,616 & 100.00 & 7,632 & 100.00 \\
\hline \multicolumn{7}{|l|}{ Calliphoridae } \\
\hline Chloroprocta idiodea (Townsend, 1935) & 0 & 0.00 & 2 & 0.10 & 2 & 0.03 \\
\hline Chrysomya albiceps (Wiedemann, 1819)* & 4,351 & 72.99 & 1,452 & 71.25 & 5,803 & 72.55 \\
\hline Chrysomya megacephala (Fabricius, 1794) & 12 & 0.20 & 53 & 2.60 & 65 & 0.81 \\
\hline Chrysomya putoria (Wiedemann, 1818)* & 480 & 8.05 & 55 & 2.70 & 535 & 6.69 \\
\hline Cochliomyia hominivorax (Coquerel, 1858) & 6 & 0.10 & 0 & 0.00 & 6 & 0.08 \\
\hline Cochliomyia macellaria (Fabricius, 1775) & 1,013 & 16.99 & 404 & 19.82 & 1,417 & 17.72 \\
\hline Hemilucilia segmentaria (Fabricius, 1805)* & 9 & 0.15 & 15 & 0.74 & 24 & 0.30 \\
\hline Lucilia cuprina (Wiedemann, 1830) & 1 & 0.02 & 1 & 0.05 & 2 & 0.03 \\
\hline Lucilia eximia (Wiedemann, 1819)* & 76 & 1.28 & 52 & 2.55 & 128 & 1.60 \\
\hline Lucilia sericata (Meigen, 1826) & 13 & 0.22 & 4 & 0.20 & 17 & 0.21 \\
\hline Total Calliphoridae & 5,961 & 100.00 & 2,038 & 100.00 & 7,999 & 100.00 \\
\hline \multicolumn{7}{|l|}{ Fanniidae } \\
\hline Fannia (subgroup pusio)* & 49 & 9.65 & 61 & 12.18 & 110 & 10.90 \\
\hline Fannia pusio (Wiedemann, 1830)* & 314 & 61.81 & 346 & 69.06 & 660 & 65.41 \\
\hline Fannia snyderi (Seago, 1954) & 90 & 17.72 & 13 & 2.59 & 103 & 10.21 \\
\hline Fannia sp. & 55 & 10.83 & 81 & 16.17 & 136 & 13.48 \\
\hline Total Fanniidae & 508 & 100.00 & 501 & 100.00 & 1,009 & 100.00 \\
\hline Phoridae * & 13 & 0.01 & 1 & 0.01 & 14 & 0.01 \\
\hline \multicolumn{7}{|l|}{ Stratiomyidae } \\
\hline Hermetia illuscens (Linnaeus, 1758)* & 38 & 0.03 & 15 & 0.08 & 53 & 0.04 \\
\hline Other Diptera & 17,951 & 16.26 & 7,831 & 41.58 & 25,769 & 19.94 \\
\hline Cotal Diptera & 110,408 & 100.00 & 18,832 & 100.00 & 129,240 & 100.00 \\
\hline
\end{tabular}


Table I. Continued.

Taxa

Dry period

Coleoptera

Cleridae

Necrobia rufipes (De Geer, 1775)*

Dermestidae

Dermestes maculatus (DeGeer, 1774)*

2,185

100.00

136

100.00

2,321

100.00

Staphylinidae

Aleochara sp.

Atheta sp.

Cryptobium sp.

Dybelonetes hibridus (Erichson, 1840)

Eulissus calybaeus (Mannerheim, 1830)

Heterothops spp.

Neophyrus sp.

Oxytellus sp.

Philonthus flavolimbatus (Erichson, 1840)

4,610

100.00

264

100.00

4,874

100.00

Philonthus spp

Other Staphylinidae

Total Staphylinidae

$22 \quad 1.76$

$37 \quad 2.96$

$1 \quad 0.08$

$9 \quad 0.72$

10.08

$10 \quad 0.80$

$2 \quad 0.16$

$1 \quad 0.08$

$723 \quad 57.75$

$417 \quad 33.31$

$29 \quad 2.32$

$1,252 \quad 100.00$

Histeridae

Histeridae spp

Total Histeridae

Scarabaeidae

Ataenius aequalis Harold, 1880

Ataenius sp.

Ateuchus vividus (Germar, 1824)

Canthidium sp.

Canthon virens (Mannerheim, 1829)

Coprophanaeus ensifer (Germar, 1824)

Deltochilum (Deltohyboma) sp.

Dichotomius nisus (Olivier, 1789)

Dichotomius opacipennis (Luederwaldt, 1931)

Eurysternus aeneus Génier, 2009

Labarrus pseudolividus (Balthasar, 1941)

Ontherus appendiculattus (Mannerheim, 1829)

Onthophagus hirculus (Mannerheim, 1829)

$156 \quad 11.89$

11.89

163

$1,312 \quad 100.00$

$18 \quad 3.16$

$40 \quad 2.20$

$\begin{array}{llll}40 & 7.03 & 77 & 4.23\end{array}$

$\begin{array}{llll}0 & 0.00 & 1 & 0.06\end{array}$

$\begin{array}{llll}9 & 1.58 & 18 & 0.99\end{array}$

$\begin{array}{llll}2 & 0.35 & 3 & 0.17\end{array}$

$\begin{array}{llll}6 & 1.05 & 16 & 0.88\end{array}$

$\begin{array}{llll}6 & 0.18 & 3 & 0.17\end{array}$

$\begin{array}{llll}0 & 0.00 & 1 & 0.06\end{array}$

$\begin{array}{rrrr}0 & 5.00 & & \\ 40 & 59.75 & 1,063 & 58.37\end{array}$

$\begin{array}{llll}132 & 23.20 & 549 & 30.15\end{array}$

$\begin{array}{llll}21 & 3.69 & 50 & 2.75\end{array}$

$\begin{array}{llll}569 & 100.00 & 1,821 & 100.00\end{array}$

Trichillum externepunctatum Preudhomme de

Scarabaeidae spp.

Total Scarabaeidae

$48 \quad 12.28$

12.28

1.54

6.65

0.51

3.07

0.00

8.44

0.25

0.00

0.51

0.00

7.88

22.76

14.07

$946 \quad 85.30$

$2,102 \quad 86.82$

1,109

14.70

$\begin{array}{rr}319 & 13.18 \\ 2,421 & 100.00\end{array}$

Geotrupidae

Bolbapium striatopunctatum (Castelnau, 1840)

Silphidae

Oxyletrum discicolle (Brullé)

Trogidae

Omorgus suberosus (Fabricius, 1775)

Other Coleoptera

tera 1,111

Total Coleoptera

1,11

13.42

\begin{tabular}{rrrrrr}
1,111 & 13.42 & 844 & 14.47 & 1,955 & 13.86 \\
\hline 8,276 & 100.00 & 5,832 & 100.00 & 14,108 & 100.00 \\
\hline
\end{tabular}

$\begin{array}{rr}53 & 16.83 \\ 3 & 0.95 \\ 42 & 13.33\end{array}$

$101 \quad 14.31$

$9 \quad 1.27$

$68 \quad 9.63$

20.28

$20 \quad 2.83$

$\begin{array}{llll}8 & 2.54 & 20 & 2.83\end{array}$

$\begin{array}{llll}2 & 0.64 & 2 & 0.28\end{array}$

$\begin{array}{llll}18 & 5.71 & 51 & 7.22\end{array}$

$\begin{array}{rrrr}18 & 5.71 & 51 & 7.22 \\ 0 & 0.00 & 1 & 0.14\end{array}$

$\begin{array}{llll}1 & 0.32 & 1 & 0.14\end{array}$

$\begin{array}{rrrr}1 & 0.32 & 1 & 0.14 \\ 12 & 3.81 & 14 & 1.98\end{array}$

$\begin{array}{llll}1 & 0.32 & 1 & 0.14\end{array}$

$\begin{array}{rrrr}1 & 0.32 & 1 & 0.14 \\ 32 & 10.16 & 141 & 19.97\end{array}$

$\begin{array}{llll}42 & 13.33 & 131 & 18.55\end{array}$

$\begin{array}{llll}98 & 31.11 & 153 & 21.67\end{array}$

\begin{tabular}{rrrrrr}
8 & 2.05 & 3 & 0.95 & 11 & 1.56 \\
\hline 391 & 100.00 & 315 & 100.00 & 706 & 100.00
\end{tabular}

$\begin{array}{llllll}2 & 100.00 & 0 & 0.00 & 2 & 100.00\end{array}$

$\begin{array}{llllll}2 & 100.00 & 1 & 100.00 & 3 & 100.00\end{array}$


Table I. Continued

\begin{tabular}{|c|c|c|c|c|c|c|c|}
\hline & \multirow{2}{*}{ Taxa } & \multicolumn{2}{|c|}{ Dry period } & \multicolumn{2}{|c|}{ Humid period } & \multicolumn{2}{|c|}{ Total } \\
\hline & & $\mathrm{AF}$ & $\%$ & $\mathrm{AF}$ & $\%$ & $\mathrm{AF}$ & $\%$ \\
\hline \multicolumn{8}{|c|}{ Other Arthropoda } \\
\hline Hemiptera & & 9,318 & 100.00 & 532 & 100.00 & 9,850 & 100.00 \\
\hline Hymenoptera & & 3,682 & 100.00 & 659 & 100.00 & 4,341 & 100.00 \\
\hline Lepidoptera & & 841 & 100.00 & 2,338 & 100.00 & 3,179 & 100.00 \\
\hline Blattodea & & 1 & 0.00 & 5 & 0.02 & 6 & 0.01 \\
\hline Dermaptera & & 0 & 0.00 & 2 & 0.01 & 2 & 0.00 \\
\hline Homoptera & & 1 & 0.00 & 1 & 0.00 & 2 & 0.00 \\
\hline Isoptera & & 47 & 0.04 & 20 & 0.07 & 67 & 0.04 \\
\hline Odonata & & 69 & 0.05 & 15 & 0.05 & 84 & 0.05 \\
\hline Orthoptera & & 159 & 0.12 & 32 & 0.11 & 191 & 0.12 \\
\hline Acari & & 13 & 0.01 & 11 & 0.04 & 24 & 0.02 \\
\hline Araneida & & 21 & 0.02 & 1 & 0.00 & 22 & 0.01 \\
\hline Total & & 132,836 & 100.00 & 28,280 & 100.00 & 161,116 & 100.00 \\
\hline
\end{tabular}

considerable abundance but only in the dry period of the year. On the other hand, Cyrtoneurina sp., Pseudoptiloleps nigripoda Snyder (Muscidae) and Micropezidae were observed exclusively in the humid period. Specimens of Microcerella erythropyga Lopes (Diptera, Sarcophagidae) were collected only at the "Campo sujo" vegetation profile (Table II).

The Coleoptera collected belong to at least 50 species of 21 families. Only Dermestes maculatus De Geer (Dermestidae) and Necrobia rufipes De Geer (Cleridae), however, were observed breeding in the carcasses. Some Coleoptera and representatives of other orders, showed preference for specific areas and periods of the year (Tables I and II). Dermestes maculatus was found in the carcasses in the second and third stages, between the third and sixth day of the postmortem interval (PMI), and their larvae were encountered on the trays under the carcasses from the sixth day of PMI (third stage) during the humid period. Adults of Necrobia rufipes were found in the carcasses from the sixth to the tenth days of PMI in both humid and dry periods. There were some adults that had emerged from larvae collected on the eleventh day of PMI, in the dry period. Both of the above species presented higher numbers of larvae during stage five of the dry period and a significant reduction in the numbers of attracted and reared adults during the humid period of the year.

Among the Scarabeidae, the following species were dominant: Trichillum externepunctatum Predhomme de Borre Onthophagus hirculus (Mannerheim), Ontherus appendiculatus (Mannerheim) and Ataenius aequalis Harold. All of these were collected during both periods of the year in both areas (Tables I and II). Coprophanaeus ensifer (Germar) (Scarabaeidae) was collected from the thirteenth day of PMI, only in the "Cerrado stricto sensu", during the humid period. Nevertheless, orifices resembling those produced by this species were observed on the soil surface beside the cages in both areas of collection.

\section{DISCUSSION}

Climatic conditions registered during the experiments were consistent with those predicted for the area: there was a dry, cool period from May to September and a rainy, hot period from October to April (Rosa et al. 1991). The greater quantity of arthropods collected in the dry period, mainly representatives of the orders Diptera and Coleoptera, seems to be related to a reduction in the rapidity of the decaying process in the carcasses during this period. The low moisture and pluviosity would have promoted more rapid dehydration, restricting colonization by the breeding fauna to a shorter period of time. On the other hand, the carcasses functioned as sources of attraction to adult insects for a longer time during the dry period (Carvalho \& Linhares 2001; Rosa et al. 2009).

The results highlight a great diversity of Sarcophagidae in the study area. Barros et al. (2008) also found great diversity among these Diptera attracted to pig carrion in Cerrado vegetation in the region of Brasília, $435 \mathrm{~km}$ from the city of Uberlândia. The genus Oxysarcodexia (Sarcophagidae) was the most diverse of the family (Table I). Similar results were also produced by Barbosa et al. (2009) in Rio de Janeiro. According to Lopes (1973), species of Oxysarcodexia prefer feces as breeding media. The presence of these flies in decaying carcasses indicates that they are using carrion as a source of protein for development of their ovocites and/or as mating sites (Archer \& Elgar 2003).

Chrysomya albiceps (Wiedemann) was the most abundant species among the Calliphoridae. Rosa et al. 2009 also found this species to be the most abundant among the breeding Diptera in pig carcass in this area. Many studies carried out in Brazil have found this species to be a very important forensic indicator (Souza \& Linhares 1997; Carvalho \& Linhares 2001; Barbosa et al. 2010), including research con- 
Table II. Absolute (AF) and relative (\%) frequencies of arthropods attracted to pig carrion (Sus scrofa L.) in two vegetation profiles of Cerrado, "Campo sujo" and "Cerrado stricto sensu", during the dry and the humid periods of the year in Uberlândia, State of Minas Gerais.

\begin{tabular}{|c|c|c|c|c|c|c|}
\hline \multirow{2}{*}{ Taxa } & \multicolumn{2}{|c|}{ Campo sujo } & \multicolumn{2}{|c|}{ Cerrado stricto sensu } & \multicolumn{2}{|c|}{ Total } \\
\hline & $\mathrm{AF}$ & $\%$ & $\mathrm{AF}$ & $\%$ & $\mathrm{AF}$ & $\%$ \\
\hline \multicolumn{7}{|l|}{ Diptera } \\
\hline \multicolumn{7}{|l|}{ Sarcophagidae } \\
\hline Argoravinia rufiventris & 13 & 0.03 & 13 & 0.04 & 26 & 0.03 \\
\hline Blaesoxipha (A.) lanei & 811 & 1.56 & 719 & 2.08 & 1,530 & 1.76 \\
\hline Blaesoxipha (A.) minensis & 16 & 0.03 & 9 & 0.03 & 25 & 0.03 \\
\hline Dexosarcophaga carvalhoi & 3,283 & 6.30 & 2,189 & 6.32 & 5,472 & 6.31 \\
\hline Dexosarcophaga paulistana & 1 & 0.00 & 0 & 0.00 & 1 & 0.00 \\
\hline Dexosarcophaga spp. & 256 & 0.49 & 138 & 0.40 & 394 & 0.45 \\
\hline Dexosarcophaga transita & 218 & 0.42 & 190 & 0.55 & 408 & 0.47 \\
\hline Helicobia aurescens & 7 & 0.19 & 76 & 0.22 & 173 & 0.20 \\
\hline Helicobia borgmeieri & 3 & 0.01 & 0 & 0.00 & 3 & 0.00 \\
\hline Helicobia morionella & 16 & 0.03 & 19 & 0.06 & 35 & 0.04 \\
\hline Helicobia pilifera & 29 & 0.06 & 44 & 0.13 & 73 & 0.08 \\
\hline Helicobia rapax & 114 & 0.22 & 73 & 0.21 & 187 & 0.22 \\
\hline Microcerella erythropyza & 19 & 0.04 & 0 & 0.00 & 19 & 0.02 \\
\hline Oxysarcodexia admixta & 72 & 0.14 & 69 & 0.20 & 141 & 0.16 \\
\hline Oxysarcodexia amorosa & 0 & 0.00 & 3 & 0.01 & 3 & 0.00 \\
\hline Oxysarcodexia angrensis & 121 & 0.23 & 220 & 0.63 & 341 & 0.39 \\
\hline Oxysarcodexia aura & 446 & 0.86 & 226 & 0.65 & 672 & 0.76 \\
\hline Oxysarcodexia avuncula & 6,502 & 12.47 & 5,391 & 15.56 & 11,893 & 13.71 \\
\hline Oxysarcodexia carvalhoi & 1 & 0.00 & 32 & 0.09 & 33 & 0.04 \\
\hline Oxysarcodexia culminiforceps & 5 & 0.01 & 5 & 0.01 & 10 & 0.01 \\
\hline Oxysarcodexia diana & 492 & 0.94 & 439 & 1.27 & 931 & 1.07 \\
\hline Oxysarcodexia eberti & 26 & 0.05 & 6 & 0.02 & 32 & 0.04 \\
\hline Oxysarcodexia fluminensis & 132 & 0.25 & 133 & 0.38 & 265 & 0.31 \\
\hline Oxysarcodexia modesta & 1 & 0.00 & 0 & 0.00 & 1 & 0.00 \\
\hline Oxysarcodexia paulistanensis & 30 & 0.06 & 3 & 0.01 & 33 & 0.04 \\
\hline Oxysarcodexia riograndensis & 5 & 0.01 & 0 & 0.00 & 5 & 0.01 \\
\hline Oxysarcodexia simplicoides & 253 & 0.48 & 176 & 0.51 & 429 & 0.49 \\
\hline Oxysarcodexia spp. & 4 & 0.01 & 16 & 0.05 & 20 & 0.02 \\
\hline Oxysarcodexia terminalis & 1 & 0.00 & 0 & 0.00 & 1 & 0.00 \\
\hline Oxysarcodexia thornax & 25,761 & 49.42 & 14,768 & 42.63 & 40,529 & 46.71 \\
\hline Oxysarcodexia xanthosoma & 0 & 0.00 & 3 & 0.01 & 3 & 0.00 \\
\hline Peckia (Euboettcheria) anguilla & 104 & 0.20 & 52 & 0.15 & 156 & 0.18 \\
\hline Peckia (Euboettcheria) australis & 0 & 0.00 & 2 & 0.01 & 2 & 0.00 \\
\hline Peckia (Euboettcheria) collusor & 114 & 0.22 & 175 & 0.51 & 289 & 0.33 \\
\hline Peckia (Euboettcheria) florencioi & 48 & 0.09 & 62 & 0.18 & 110 & 0.13 \\
\hline Peckia (Pattonella) intermutans & 40 & 0.08 & 5 & 0.01 & 45 & 0.05 \\
\hline Peckia (Peckia) chrysostoma & 57 & 0.11 & 28 & 0.08 & 85 & 0.10 \\
\hline Peckia (Peckia) pexata & 64 & 0.12 & 11 & 0.03 & 75 & 0.09 \\
\hline Peckia (Squamatodes) ingens & 7 & 0.01 & 1 & 0.00 & 8 & 0.01 \\
\hline Peckia (Squamatodes) trivitatta & 200 & 0.38 & 95 & 0.27 & 295 & 0.34 \\
\hline Ravinia advena & 327 & 0.63 & 85 & 0.25 & 412 & 0.47 \\
\hline Ravinia belforti & 1,068 & 2.05 & 937 & 2.70 & 2,005 & 2.31 \\
\hline Sarcodexia lambens & 527 & 1.01 & 839 & 2.42 & 1,37 & 1.57 \\
\hline Sarconeiva fimbriata & 1 & 0.00 & 0 & 0.00 & 1 & 0.00 \\
\hline Sarcophaga (L.) crispula & 87 & 0.17 & 39 & 0.11 & 126 & 0.15 \\
\hline Titanogrypa (C.) larvicida & 80 & 0.15 & 6 & 0.02 & 86 & 0.10 \\
\hline
\end{tabular}


Table II. Continued.

\begin{tabular}{|c|c|c|c|c|c|c|}
\hline \multirow{2}{*}{ Taxa } & \multicolumn{2}{|c|}{ Campo sujo } & \multicolumn{2}{|c|}{ Cerrado stricto sensu } & \multicolumn{2}{|c|}{ Total } \\
\hline & $\mathrm{AF}$ & $\%$ & $\mathrm{AF}$ & $\%$ & $\mathrm{AF}$ & $\%$ \\
\hline Tricharaea (S.) occidua & 3,358 & 6.44 & 2,850 & 8.23 & 6,208 & 7.15 \\
\hline Tricharaea (S.) ramirezi & 15 & 0.03 & 13 & 0.04 & 28 & 0.03 \\
\hline Sarcophagidae spp. & 7,297 & 14.00 & 4,482 & 12.94 & 11,779 & 13.58 \\
\hline Total Sarcophagidae & 52,122 & 100.00 & 34,642 & 100.00 & 86,764 & 100.00 \\
\hline \multicolumn{7}{|l|}{ Muscidae } \\
\hline Atherigona orientalis & 226 & 4.76 & 816 & 28.28 & 1,042 & 13.65 \\
\hline Biopyrellia bipuncta & 259 & 5.46 & 597 & 20.69 & 856 & 11.22 \\
\hline Brontaea debilis & 116 & 2.44 & 19 & 0.66 & 135 & 1.77 \\
\hline Brontaea spp. & 1 & 0.02 & 2 & 0.07 & 3 & 0.04 \\
\hline Cyrtoneurina sp. & 5 & 0.11 & 6 & 0.21 & 11 & 0.14 \\
\hline Cyrtoneuropsis conspersa & 1 & 0.02 & 0 & 0.00 & 1 & 0.01 \\
\hline Cyrtoneuropsis rescita & 479 & 10.09 & 215 & 7.45 & 694 & 9.09 \\
\hline Graphomya maculata & 27 & 0.57 & 8 & 0.28 & 35 & 0.46 \\
\hline Myospila sp. & 2 & 0.04 & 0 & 0.00 & 2 & 0.03 \\
\hline Morellia ochricornis & 15 & 0.32 & 21 & 0.73 & 36 & 0.47 \\
\hline Musca domestica & 1,417 & 29.85 & 510 & 17.68 & 1,927 & 25.25 \\
\hline Ophyra aenescens & 1,970 & 41.50 & 567 & 19.65 & 2,537 & 33.24 \\
\hline Ophyra chalcogaster & 2 & 0.04 & 11 & 0.38 & 13 & 0.17 \\
\hline Pseudoptilolepis nigripoda & 6 & 0.13 & 8 & 0.28 & 14 & 0.18 \\
\hline Sarcopromusca pruna & 175 & 3.69 & 98 & 3.40 & 273 & 3.58 \\
\hline Stomoxys calcitrans & 44 & 0.93 & 7 & 0.24 & 51 & 0.67 \\
\hline Synthesiomyia nudiseta & & 0.04 & 0 & 0.00 & 2 & 0.03 \\
\hline Total Muscidae & 4,747 & 100.00 & 2,885 & 100.00 & 7,632 & 100.00 \\
\hline \multicolumn{7}{|l|}{ Calliphoridae } \\
\hline Chloroprocta idiodea & 0 & 0.00 & 2 & 0.06 & 2 & 0.03 \\
\hline Chrysomya albiceps & 3,467 & 71.62 & 2,336 & 73.97 & 5,803 & 72.55 \\
\hline Chrysomya megacephala & 23 & 0.48 & 42 & 1.33 & 65 & 0.81 \\
\hline Chrysomya putoria & 259 & 5.35 & 276 & 8.74 & 535 & 6.69 \\
\hline Cochliomyia hominivorax & 6 & 0.12 & 0 & 0.00 & 6 & 0.08 \\
\hline Cochliomyia macellaria & 1,000 & 20.66 & 417 & 13.21 & 1,417 & 17.72 \\
\hline Hemilucilia segmentaria & 19 & 0.39 & 5 & 0.16 & 24 & 0.30 \\
\hline Lucilia cuprina & 1 & 0.02 & 1 & 0.03 & 2 & 0.03 \\
\hline Lucilia eximia & 61 & 1.26 & 67 & 2.12 & 128 & 1.60 \\
\hline Lucilia sericata & 5 & 0.10 & 12 & 0.40 & 17 & 0.21 \\
\hline Total Calliphoridae & 4,841 & 100.00 & 3,158 & 100.00 & 7,999 & 100.00 \\
\hline \multicolumn{7}{|l|}{ Fanniidae } \\
\hline Fannia (subgroup pusio) & 84 & 12.37 & 26 & 7.88 & 110 & 10.90 \\
\hline Fannia pusio & 418 & 61.56 & 242 & 73.33 & 660 & 65.41 \\
\hline Fannia snyderi & 80 & 11.78 & 23 & 6.97 & 103 & 10.21 \\
\hline Fannia sp. & 97 & 14.29 & 39 & 11.82 & 136 & 13.48 \\
\hline Total Fanniidae & 679 & 100.00 & 330 & 100.00 & 1,009 & 100.00 \\
\hline Phoridae & 3 & 0.00 & 11 & 0.02 & 14 & 0.01 \\
\hline Hermetia illuscens & 38 & 0.05 & 15 & 0.03 & 53 & 0.04 \\
\hline Other Diptera & 15,640 & 20.03 & 10,129 & 19.79 & 25,769 & 19.94 \\
\hline Total Diptera & 78,070 & 100.00 & 51,170 & 100.00 & 129,240 & 100.00 \\
\hline \multicolumn{7}{|l|}{ Coleoptera } \\
\hline \multicolumn{7}{|l|}{ Staphylinidae } \\
\hline Aleochara sp. & 16 & 1.46 & 24 & 3.30 & 40 & 2.20 \\
\hline
\end{tabular}


Table II. Continued.

\begin{tabular}{|c|c|c|c|c|c|c|}
\hline \multirow{2}{*}{ Taxa } & \multicolumn{2}{|c|}{ Campo sujo } & \multicolumn{2}{|c|}{ Cerrado stricto sensu } & \multicolumn{2}{|c|}{ Total } \\
\hline & $\mathrm{AF}$ & $\%$ & $\mathrm{AF}$ & $\%$ & $\mathrm{AF}$ & $\%$ \\
\hline Atheta $\mathrm{sp}$. & 35 & 3.20 & 42 & 5.78 & 77 & 4.23 \\
\hline Cryptobium sp. & 1 & 0.09 & 0 & 0.00 & 1 & 0.06 \\
\hline Dybelonetes hibridus & 9 & 0.82 & 9 & 1.24 & 18 & 0.99 \\
\hline Eulissus calybaeus & 1 & 0.09 & 2 & 0.27 & 3 & 0.17 \\
\hline Heterothops sp. & 10 & 0.91 & 6 & 0.83 & 16 & 0.88 \\
\hline Neophyrus sp. & 2 & 0.18 & 1 & 0.14 & 3 & 0.17 \\
\hline Oxytellus sp. & 1 & 0.09 & 0 & 0.00 & 1 & 0.06 \\
\hline Philonthus flavolimbatus & 595 & 54.39 & 468 & 64.37 & 1,063 & 58.37 \\
\hline Philonthus spp. & 398 & 36.38 & 151 & 20.77 & 549 & 30.15 \\
\hline Staphylinidae sp. & 26 & 2.38 & 24 & 3.30 & 50 & 2.75 \\
\hline Total Staphylinidae & 1,094 & 100.00 & 727 & 100.00 & 1,821 & 100.00 \\
\hline \multicolumn{7}{|l|}{ Dermestidae } \\
\hline Dermestes maculatus & 2,129 & 100.00 & 2,745 & 100.00 & 4,874 & 100.00 \\
\hline \multicolumn{7}{|l|}{ Cleridae } \\
\hline Necrobia rufipes & 879 & 100.00 & 1,442 & 100.00 & 2,321 & 100.00 \\
\hline \multicolumn{7}{|l|}{ Histeridae } \\
\hline Euspilotus spp. & 1,038 & 87.74 & 1,064 & 85.95 & 2,102 & 86.82 \\
\hline Histeridae spp. & 145 & 12.26 & 174 & 14.06 & 319 & 13.18 \\
\hline Total Histeridae & 1,183 & 100.00 & 1,238 & 100.00 & 2,421 & 100.00 \\
\hline \multicolumn{7}{|l|}{ Scarabaeidae } \\
\hline Ataenius aequalis & 48 & 11.77 & 53 & 17.78 & 101 & 14.31 \\
\hline Ataenius sp. & 5 & 1.22 & 4 & 1.34 & 9 & 1.27 \\
\hline Ateuchus vividus & 21 & 5.15 & 47 & 15.77 & 68 & 9.63 \\
\hline Canthidium sp. & 2 & 0.49 & 0 & 0.00 & 2 & 0.28 \\
\hline Canthon virens & 18 & 4.41 & 2 & 0.67 & 20 & 2.83 \\
\hline Coprophanaeus ensifer & 0 & 0.00 & 2 & 0.67 & 2 & 0.28 \\
\hline Deltochilum (Deltohyboma) sp. & 20 & 4.90 & 31 & 10.40 & 51 & 7.22 \\
\hline Dichotomius nisus & 1 & 0.25 & 0 & 0.00 & 1 & 0.14 \\
\hline Dichotomius opacipennis & 0 & 0.00 & 1 & 0.34 & 1 & 0.14 \\
\hline Eurysternus aff. hirtellus & 1 & 0.25 & 13 & 4.36 & 14 & 1.98 \\
\hline Labarrus pseudolividus & 0 & 0.00 & 1 & 0.34 & 1 & 0.14 \\
\hline Ontherus appendiculattus & 122 & 29.90 & 19 & 6.38 & 141 & 19.97 \\
\hline Onthophagus hirculus & 107 & 26.23 & 24 & 8.05 & 131 & 18.56 \\
\hline Trichillum externepunctatum & 55 & 13.48 & 98 & 32.89 & 153 & 21.67 \\
\hline Scarabaeidae spp. & 8 & 1.96 & 3 & 1.01 & 11 & 1.56 \\
\hline Total Scarabaeidae & 408 & 100.00 & 298 & 100.00 & 706 & 100.00 \\
\hline \multicolumn{7}{|l|}{ Bolboceratidae } \\
\hline Bolbapium striatopunctatum & 2 & 100.00 & 0 & 0.00 & 2 & 100.00 \\
\hline \multicolumn{7}{|l|}{ Silphidae } \\
\hline Oxyletrum disciolle & 1 & 100.00 & 2 & 100.00 & 3 & 100.00 \\
\hline \multicolumn{7}{|l|}{ Trogidae } \\
\hline Omorgus suberosus & 3 & 100.00 & 2 & 100.00 & 5 & 100.00 \\
\hline Other Coleoptera & 820 & 12.58 & 1,135 & 14.96 & 1,955 & 13.86 \\
\hline Total Coleoptera & 6,519 & 100.00 & 7,589 & 100.00 & 14,108 & 100.00 \\
\hline \multicolumn{7}{|l|}{ Other Arthropoda } \\
\hline Hemiptera & 5,496 & 5.85 & 4,354 & 6.48 & 9,850 & 6.11 \\
\hline Hymenoptera & 2,495 & 2.56 & 1,846 & 2.75 & 4,341 & 2.69 \\
\hline
\end{tabular}


Table II. Continued.

\begin{tabular}{|c|c|c|c|c|c|c|}
\hline \multirow{2}{*}{ Taxa } & \multicolumn{2}{|c|}{ Campo sujo } & \multicolumn{2}{|c|}{ Cerrado stricto sensu } & \multicolumn{2}{|c|}{ Total } \\
\hline & $\mathrm{AF}$ & $\%$ & $\mathrm{AF}$ & $\%$ & $\mathrm{AF}$ & $\%$ \\
\hline Lepidoptera & 1,234 & 1.31 & 1,945 & 2.90 & 3,179 & 1.97 \\
\hline Blattodea & 2 & 0.00 & 4 & 0.01 & 6 & 0.00 \\
\hline Dermaptera & 2 & 0.00 & 0 & 0.00 & 2 & 0.00 \\
\hline Homoptera & 1 & 0.00 & 1 & 0.00 & 2 & 0.00 \\
\hline Isoptera & 0 & 0.000 & 67 & 0.100 & 67 & 0.04 \\
\hline Odonata & 25 & 0.03 & 59 & 0.09 & 84 & 0.05 \\
\hline Orthoptera & 87 & 0.09 & 104 & 0.16 & 19 & 0.12 \\
\hline Acari & 11 & 0.01 & 13 & 0.02 & 24 & 0.02 \\
\hline Araneida & 10 & 0.01 & 12 & 0.02 & 22 & 0.01 \\
\hline Total & 93,952 & 100.00 & 67,164 & 100.00 & 161,116 & 100.00 \\
\hline
\end{tabular}

ducted in areas of the Cerrado (Marchiori et al. 2000; Rosa et al. 2009). Cochliomyia macellaria Fabricius was the second most numerous species among the Calliphoridae attracted to the carrion. This result was also observed by Souza \& Linhares (1997) in the State of São Paulo. The introduction of Chrysomya species on the American Continent has interfered with the distribution of $C$. macellaria in many areas (Boumgartner \& Greenberg 1984). Nevertheless, recent research developed in Rio de Janeiro e Rio Grande do Norte has documented the collection of immature individuals and adults of this species in human cadavers, suggesting a reduced impact of exotic species upon it in those localities (Oliveira-Costa \& Mello-Patiu 2004; Andrade et al. 2005).

Coleoptera, mainly Staphylinidae and Scarabaeidae, presented a different seasonal distribution from Diptera and manifested greater diversity of species during the humid period. Campobasso et al. (2001) identified temperature as an important factor affecting the quantity and diversity of insects associated with decaying carcasses. Temperature and humidity appeared to be the principal factors responsible for the seasonal variations of Coleoptera, mostly Staphylinidae and Scarabaeidae, in the present study. Staphylinidae and Histeridae occurred from the second to the fifth stages of decay. They are predators on the immature insects growing in the carcasses during the process of decomposition.

Dermestidae are pests that feed on dry organic materials (Schroeder et al. 2002). Carcasses in the final stages of decay can be used by them as a food source for the adults and immature stages (Souza \& Linhares 1997; Schroeder et al. 2002; Carvalho et al. 2004; Velásquez 2008). They remove the remains of muscles and tendons (Hefti et al. 1980). Omorgus suberosus (Fabricius) (Trogidae) is another species that feeds on dry organic remains rich in nitrogen, such as animal carcasses and bird excrement (FZVM, pers. obs.). Necrobia rufipes (De Geer) has also been encountered colonizing partially or totally dry human cadavers and animal carcasses in Brazil (Souza \& Linhares 1997; Carvalho et al. 2004; Almeida \& Mise 2009). The decrease in the numbers of $D$. maculatus during the humid period is probably related to the concomitant decrease in the duration of the final stages of the decaying process during this period. The greater humidity also reduces the attractiveness of the carcasses for these Coleoptera.

Studies conducted by Marchiori et al. (2000) in an area of Cerrado close to the town of Itumbiara, in the State of Goiás, identified $T$. externepunctatum as the most abundant Scarabaeidae in decaying carcasses. This specie, as do the majority of the dominant Scarabaeidae in this study, breeds in the excrement of herbivorous mammals and its presence in the carcasses is related to feeding but without nidification activity. Its larvae, as well as those of Ataenius aequalis, breed directly in their food source (López-Alarcón et al. 2009). Carvalho et al. (2000) encountered Coprophanaeus ensifer (Germar) associated with carcasses in a forested area in the State of São Paulo and described it as an important PMI and localization indicator. Coprophanaeus ensifer and Deltochilum (Deltohyboma) sp. were the Scarabaeidae strictly or mostly necrophagous encountered in the present study (FZVM, per. obs.). The trays placed in the bottoms of the cages containing the carcasses may have interfered with the underground nidification of these species and also the nidification of other Scarabaeinae (Halffter \& Edmonds 1982).

It is possible, however, that the trays were not deep enough to retain all the ambulatory Arthrododa species and postfeeding larvae. As a result, part of them may not have been properly sampled. Although many of the species registered here are potential indicators of seasonality, the possibility of using the majority of them that do not breed in carcasses as indicators is restricted when compared with those species that also breed in the substrate. On the other hand, the results presented, associated with those that can be obtained from the anthropic environments - areas of urban and agricultural and cattle-raising activities - may identify some the species registered here as indicators of specific natural areas.

The occurrence of an entomological succession throughout the decaying process of the carcasses was clearly observed. Despite the constant presence of insects, some species/groups occurred in the carcasses only in certain decaying stages. The 
present study demonstrates a great diversity of entomofauna associated with carrion in an ecosystem rarely studied until now. Considering the Cerrado as a biome with great biodiversity and high rates of endemism but largely unknown (Ribeiro et al. 1983), the information presented is significant from an ecological point of view and also for medico-legal forensic entomology.

\section{ACKNOWLEDGEMENT}

The authors wish to thank Marcia Souto Couri (Museu Nacional, Universidade Federal do Rio de Janeiro), for her assistance in identifying the Muscidae. Fernando Z. Vaz-deMello is CNPq fellow (PQ 304925/2010-1). Thiago A. Rosa was CAPES fellow from 2005 to 2007.

\section{REFERENCES}

Almeida, L. M. \& K. M. Mise. 2009. Diagnosis and Key of the main families and species of South American Coleoptera of forensic importance. Revista Brasileira de Entomologia 53: 227-244

Andrade, H. T. A.; A. A. Varela-Freire; M. J. A. Batista. \& J. F. Medeiros. 2005. Calliphoridae (Diptera) Coletados em Cadáveres Humanos no Rio Grande do Norte. Neotropical Entomology 34: 855-856.

Archer, M. S. \& M. A. Elgar. 2003. Effects of decomposition on carcass attendance in a guild of carrion-breeding flies. Medical and Veterinary Entomology 17: 263-271.

Archer, M. S.; R. B. Bassed; C. A. Briggs \& M. J. Lynch. 2005. Social isolation and delayed discovery of bodies in houses: The value of forensic pathology, anthropology, odontology and entomology in the medico-legal investigation. Forensic Science International 151: 259-265.

Barbosa, R. R.; C. A. Mello-Patiu; A. Ururahy-Rodrigues; C. G. Barbosa \& M. M. C. Queiroz. 2010. Temporal distribution of ten calyptrate dipteran species of medicolegal importance in Rio de Janeiro, Brazil. Memórias do Instituto Oswaldo Cruz 105: 191-198.

Barbosa, R. R.; C. A. Mello-Patiu; R. P. Mello \& M. M. C. Queiroz. 2009. New records of calyptrate dipterans (Fanniidae, Muscidae and Sarcophagidae) associated with the decomposition of domestic pigs in Brazil. Memórias do Instituto Oswaldo Cruz 104: 923-926.

Barros, R. M; C. A. Mello-Patiu \& J. R. Pujol-Luz. 2008. Sarcophagidae (Insecta, Diptera) associados à decomposição de carcaças de Sus scrofa Linnaeus (Suidae) em área de Cerrado do Distrito Federal, Brasil. Revista Brasileira de Entomologia 52: 606-609.

Bornemissza, G. F. 1957. An analysis of arthropod succession in carrion and the effect of its decomposition on the soil fauna. Australian Journal of Zoology 5: 1-12.

Boungartner, P. L \& B. Greenberg. 1984. The genus Chrysomya (Diptera: Calliphoridae) in the New World. Journal of Medical Entomology 21: 105-113.

Campobasso, C. P.; G. Di Vella \& F. Introna. 2001. Factors affecting decomposition and Diptera colonization. Forensic Science International 120: $18-27$

Carvalho, L. M. L. \& A. X. Linhares. 2001. Seasonality of insect succession and pig carcass decomposition in a natural forest area in Southeastern Brazil. Journal of Forensic Sciences 46: 604-608.

Carvalho, L.M. L., P. J. Thyssen, A. X. Linhares \& F. A. B. Palhares. 2000. A checklist of arthropods associated with pig carrion and human corpses in Southeastern Brazil. Memórias do Instituto Oswaldo Cruz 95: 135-138.
Carvalho, L. M. L; P. J. Thyssen; Goff M. L. \& A. X. Linhares. 2004. Observations on succession patterns of necrophagous insects on pig carcass in a urban area of Southeastern Brazil. Aggrawal's Internet Journal of Forensic Medicine and Toxicology 5: 40-44.

Centeno, N.; M. Maldonado \& A. Oliva. 2002. Seasonal patterns of arthropods occurring on sheltered and unsheltered pig carcasses in Buenos Aires Province (Argentina). Forensic Science International 126: $63-70$.

Halffter, G. \& W. D. Edmonds. 1982. The Nesting Behavior of Dung Beetles: An ecological and evolutive approach. Instituto de Ecología, México, D. F., 176 p.

Hanski, I. 1987. Nutritional ecology of dung-and carrion-feeding insects, p. 834-887. In: F. J. R. Slaniki \& J. G Rodrigues (eds.). Nutricional ecology of insects, mites, spiders and related invertebrates. New York, John Wiley \& Sons, Ins., 1016 p.

Hefti, E.; U. Trechsel; H. Rüfenacht \& H. Fleisch. 1980. Use of dermestid beetles for cleaning bones. Calcified Tissue Internacional 31: 45-47.

López-Alarcón, D.; G. Halffter \& F. Z. Vaz-de-Mello. 2009. Nesting Behavior in Trichillum Harold, 1868 and Related Genera (Coleoptera: Scarabaeidae: Scarabaeinae: Ateuchini: Scatimina): Primitive Process or a Loss of Nidification? The Coleopterists Bulletin 63: 289-297.

Lopes, H. S. 1973. Collecting and rearing sarcophagid flies (Diptera) in Brazil during forty years. Anais da Academia Brasileira de Ciências 30: 279-299.

Kimberly, L. T.; R. D. Fell \& C.C. Brewster. 2005. Insect fauna visiting carrion in Southwest Virginia. Forensic Science International 150: $73-80$

Marchiori, C. H.; C. G. Silva, E. R. Caldas; C. I. S. Vieira; K. G. S. Almeida; F. F. Teixeira \& A. X. Linhares. 2000. Artrópodos associados com carcaça de suíno em Itumbiara, sul de Goiás. Arquivos do Instituto de Biologia 67: 167-170

Oliveira-Costa, J. \& C. A. Mello-Patiu. 2004. Application of Forensic Entomology to estimate of the postmortem interval (PMI) in homicide investigations by the Rio de Janeiro Departament in Brazil. Aggrawal's Internet Journal of Forensic Medicine and Toxicology 5: 40-44.

Ribeiro, J. F.; S. M. Sano; J. Macedo \& J. da Silva. 1983. Os principais tipos fisionômicos da região dos cerrados. v. 21, Planaltina, Boletim de Pesquisa, EMBRAPA/CPAC, 28 p.

Rosa, T. A.; Babata, M. L. Y.; C. M. Souza; D. Sousa; C. A. Mello-Patiu \& J. Mendes. 2009. Dípteros de Interesse Forense em Dois Perfis de Vegetação de Cerrado em Uberlândia, MG. Neotropical Entomology 38 : 859-866.

Rosa, R.; S. C. Lima \& W. L. Assunção. 1991. Abordagem preliminar das condições climáticas de Uberlândia (MG). Sociedade e Natureza 3: 91108 .

Schroeder, S.; H. Klotzbach; L. Oesterhelweg \& K. Püschel. 2002. Larder beetles (Coleoptera, Dermestidae) as an accelerating factor for decomposition of a human corpse. Forensic Science International 127: $231-236$

Souza, A. M. \& A. X. Linhares. 1997. Diptera and Coleoptera of potential forensic importance in southeastern Brazil: relative abundance and seasonality. Medical and Veterinary Entomology 11: 8-12.

Thyssen, P. J.; A. C. Lessinger; A. M. L. Azeredo-Espin \& A. X. Linhares. 2005. The value of PCR-RFLP molecular markers for the differentiation of immature stages of two necrophagous flies (Diptera: Calliphoridae) of potential forensic importance, Neotropical Entomology 34: 777783.

Turchetto, M. \& S. Vanin. 2004. Forensic entomology and climatic change. Forensic Science International 146S: 207-209.

Velásquez, Y. 2008. A checklist of arthropods associated with rat carrion in a mountane locality of northern Venezuela. Forensic Science International 174: 68-70. 